Algebra Colloquium 23:4 (2016) 721-724

Algebra

Colloquium

(C) 2016 AMSS CAS

\& SUZHOU UNIV

\title{
Contents of Volume 23
}

Auslander-Reiten Sequences or Triangles Related to Rigid Subcategories

M. Lu ...

Finite Groups Whose Character Graphs Associated with Codegrees

Have No Triangles

H. Xiong

Factorizations of Matrices over Projective-free Rings

H.Y. Chen, H. Kose, Y. Kurtulmaz

Coset Diagram for the Action of Picard Group on $\mathbb{Q}(i, \sqrt{3})$

Q. Mushtaq, S. Anis

A Bijective Map on Normal Fusion Subsystems

X.Z. Xu

Graph Properties and Stratified Presentations of Partially Ordered Sets

J. Guo, T.S. Wu

On Certain Functional Equation in Semiprime Rings

N. Širovnik, J. Vukman

The Common Solution of Some Matrix Equations

L. Wang, Q.W. Wang, Z.H. He

Some Properties of Completely Arithmetical Rings

X.M. Lu

An Application of Rafael's Theorem over Partial Entwined Modules

S.X. Wang, S.J. Guo

Gorenstein Projective Modules over Triangular Matrix Rings

H. Eshraghi, R. Hafezi, Sh. Salarian, Z.W. Li

On Solvability of Groups with a Few Non-cyclic Subgroups

M. Zarrin

Quasi-morphisms via Local Action Data and Quasi-isometries

G. Ben-Simon

Isometry Groups of Some Dunwoody Manifolds

F. Spaggiari, A.I. Telloni

The Steinberg Lie Algebra $s t_{2}(S)$

Y.J. Wang, Y.Q. Shi, Y. Gao 
Envelopes and Covers by $n$-Absolutely Pure Modules

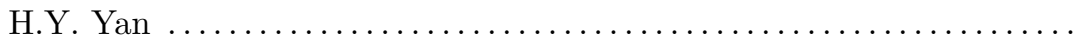

Quasi-linear Cycle Sets and the Retraction Problem for Set-theoretic

Solutions of the Quantum Yang-Baxter Equation

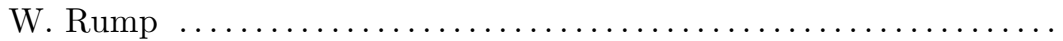

A New Expression of the Hermitian Solutions to a System of Matrix

Equations with Applications

G.J. Song, S.W. Yu, M. Chen

Groups with Finitely Many Homomorphic Images of Finite Rank

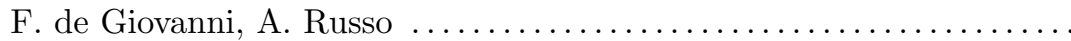

The Stable Behavior of the Augmentation Quotients of the Groups of Order $p^{5}, \mathrm{I}$

X.L. Wang

$N$-Derivations for Finitely Generated Graded Lie Algebras

H.F. Lian, C. Chen

Groups with Supersoluble Non-normal Subgroups

M. De Falco, M. Martusciello, C. Musella

A Relative Theory for Leibniz $n$-Algebras

G.R. Biyogmam

$t$-Singular Linear Spaces

J. Guo, F.G. Li, K.S. Wang

Generalized Canonical Isomorphisms on Determinant

J.K. Chen

On Direct Limits of Finite Products of Fields as Subrings of a

Commutative Ring

D. Karim

On Stability of F-Gorenstein Flat Categories

J.S. Hu, A.M. Xu

Test Elements, Generic Elements and Almost Primitivity in Free Products

A.-K. Engel, B. Fine, G. Rosenberger .......................

Connected Graphs Arising from Products of Veronese Varieties

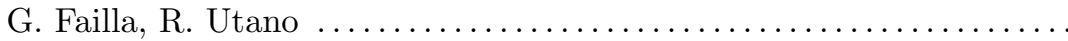

Algebraic Properties of Universal Squarefree Lexsegment Ideals

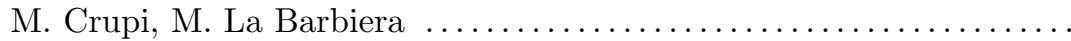

Two-parameter Quantum Superalgebras and PBW Theorem

C.R. Ai, S.L. Yang

The Influence of the Number of Non-(sub)normal Non-abelian Subgroups on Solvability of Finite Groups

J.T. Shi, C. Zhang 
Lower Bounds for Local Cohomology Modules with Respect to a Pair of Ideals

M. Lotfi Parsa, Sh. Payrovi

On Radicals of Skew Inverse Laurent Series Rings

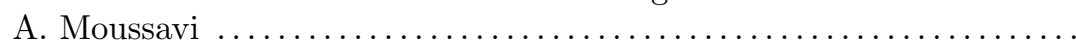

Generators of Simple Modular Lie Superalgebras

L.M. Tang, W.D. Liu

Invariant Symmetric Bilinear Forms and Derivation Algebras of Unitary

Lie Algebras

Y.L. Zheng, J.W. Gao, Z.H. Chang, Y. Gao

Derived Equivalences and Recollements of Differential Graded Algebras and Schemes

X.H. Chen, M. Lu

Some Characterizations of a Normal Subgroup of a Group and Isotopic Classes of Transversals

V. Kakkar, R.P. Shukla

Locally Graded $n$-Bell Groups

A. Faramarzi Salles

Quasitriangular Turaev Group Coalgebras and Radford's Biproduct

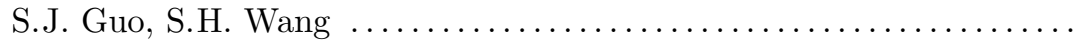

The Derivation Algebra and Automorphism Group of the Twisted $N=2$ Superconformal Algebra

H.X. Fa, J.Z. Han, X.Q. Yue

Minimal Non-nilpotent and Locally Nilpotent Fusion Systems

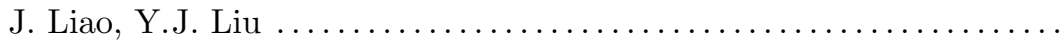

Modules with Finitely Many Submodules

S. Akbari, S.K. Ghezelahmad, E. Yaraneri

Cohen-Macaulay and Gorenstein Path Ideals of Trees

S. Saeedi Madani, D. Kiani

Co-stability of Radicals and Its Applications to PI-Theory

A.S. Gordienko

$(G, s)$-Transitive Graphs of Valency 7

S.T. Guo, Y.T. Li, X.H. Hua

On the Normality of One-fibered Monomial Ideals

C. Beddani, W. Messirdi

Maps Preserving Commutators on the Standard Borel Subgroup of the Unitary Group over a Field

H. You, X.M. Zhou 
Squarefree Monomial Modules and Extremal Betti Numbers

On Local Nilpotency of the Normal Subgroups of a Group

Z.R. Zhang, J.C. Li

Existence, Uniqueness and Functoriality of the Perfect Locality over

a Frobenius $P$-Category

L. Puig

Automorphism Groups of Some Finite $p$-Groups

H.G. Liu, Y.L. Wang

Semicomplete Finite $p$-Groups of Class 2

M. Shabani Attar

On Nilpotent Finite Alternative Rings with Planar Zero-Divisor Graphs

A.S. Kuzmina

Irreducibility Properties of Keller Maps

M. de Bondt, D. Yan

Regular Orbits of Extra-special Groups

J.E. Goodson

Homology of Powers of Ideals: Artin-Rees Numbers of Syzygies and the Golod Property

J. Herzog, V. Welker, S. Yassemi

Gelfand-Kirillov Dimensions of Modules over Differential Difference

Algebras

X.G. Zhao, Y. Zhang

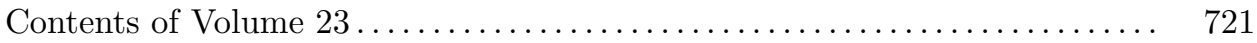

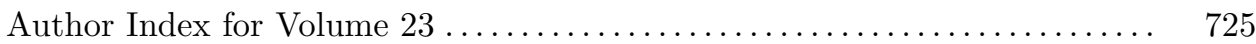

